OPEN ACCESS

Edited by:

Ding Shi,

Zhejiang University, China

Reviewed by:

Vik Meadows,

Indiana University, United States

Hua Wang,

Anhui Medical University, China

*Correspondence:

Huikuan Chu

2012xh0827@hust.edu.cn

Ling Yang

hepayang@163.com

Xiaohua Hou

houxh@hust.edu.cn

Specialty section:

This article was submitted to

Gastroenterology,

a section of the journal

Frontiers in Medicine

Received: 21 December 2021

Accepted: 31 January 2022

Published: 03 March 2022

Citation:

Chen L, Zhu Y, Hou X, Yang $L$ and Chu H (2022) The Role of Gut Bacteria and Fungi in Alcohol-Associated Liver Disease. Front. Med. 9:840752.

doi: 10.3389/fmed.2022.840752

\section{The Role of Gut Bacteria and Fungi in Alcohol-Associated Liver Disease}

\author{
Liuying Chen ${ }^{1}$, Yixin Zhu ${ }^{2}$, Xiaohua Hou ${ }^{1 *}$, Ling Yang ${ }^{1 *}$ and Huikuan Chu ${ }^{1 *}$ \\ 1 Division of Gastroenterology, Union Hospital, Tongji Medical College, Huazhong University of Science and Technology, \\ Wuhan, China, ${ }^{2}$ Department of Medicine, University of California, San Diego, San Diego, CA, United States
}

Cirrhosis and liver cancer caused by alcohol-associated liver disease (ALD) are serious threats to people's health. In addition to hepatic cell apoptosis and liver inflammation caused by oxidative stress during alcohol metabolism, intestinal microbiota disorders are also involved in the onset and development of ALD. Ethanol and its' oxidative and non-oxidative metabolites, together with dysbiosis-caused-inflammation, destroys the intestinal barrier. Changes of several microbial metabolites, such as bile acids, short-chain fatty acids, and amino acid, are closely associated with gut dysbiosis in ALD. The alcohol-caused dysbiosis can further influence intestinal barrier-related proteins, such as mucin2, bile acid-related receptors, and aryl hydrocarbon receptor (AhR), and these abnormal changes also participate in the injury of the intestinal barrier and hepatic steatosis. Gut-derived bacteria, fungi, and their toxins, such as lipopolysaccharide (LPS) and $\beta$-glucan translocate into the liver through the damaged intestinal barrier and promote the progression of inflammation and fibrosis of ALD. Thus, the prevention of alcohol-induced disruption of intestinal permeability has a beneficial effect on ALD. Currently, multiple therapeutic treatments have been applied to restore the gut microbiota of patients with ALD. Fecal microbial transplantation, probiotics, antibiotics, and many other elements has already shown their ability of restoring the gut microbiota. Targeted approaches, such as using bacteriophages to remove cytolytic Enterococcus faecalis, and supplement with Lactobacillus, Bifidobacterium, or boulardii are also powerful therapeutic options for ALD.

Keywords: gut dysbiosis, fungi, alcohol-associated liver disease, gut-liver axis, intestinal barrier

\section{INTRODUCTION}

According to the most recent WHO data, the burden of alcohol-associated liver disease (ALD) is growing (1). The natural disease course of ALD ranges from asymptomatic liver steatosis, alcoholic hepatitis to the development of cirrhosis, and liver cancer. Alcohol-mediated reactive oxygen species (ROS) formation and hepatic inflammation are the main pathophysiologies of ALD (2). Recently, the role of the gut-liver axis in ALD development and progression has attracted the attention of researchers. Germ-free mice receiving microbiota from patients with alcohol hepatitis gained more serious liver inflammation and disruption of the intestinal integrity, while the mice receiving microbiota from patients without alcoholic hepatitis could reverse the alcohol-caused liver injuries (3).

Cytotoxic effects of ROS during ethanol metabolism are the pathological basis of ALD, and the injuries are exacerbated by hypoxia, inflammation, and bacterial translocation (4). Studies about 
the role of intestinal flora in ALD have made breakthroughs in the past few years. On one hand, dysbiosis induces intestinal barrier injury, promotes lipopolysaccharide (LPS) and other pathogen-associated molecular patterns (PAMPs) translocation and aggravates inflammatory damage in the liver (5). On the other hand, dysbiosis related exotoxins, such as cytolysin from Enterococcus faecalis (6) and Candidalysin from Candida albicans (7) directly cause hepatocyte death and liver injury. Among the intestinal microorganism, bacteria and fungi are the most studied in ALD. Therefore, we review the interaction between alcohol associated liver disease and the gut microbiome and/or mycobiome to evaluate the contribution of intestinal dysbiosis to ALD. In addition, therapeutic options to restore the intestinal micro-ecosystem will be discussed.

\section{GUT-LIVER CROSS TALK}

About $70 \%$ of liver's blood supply come from the gut through the portal vein. In addition to bringing nutrients through the portal vein, there is also a chance for intestinal microbiota and their products to enter the liver, especially with increased intestinal permeability. Liver inflammation caused by PAMPs translocated from intestine is the key progression factor of ALD (8) (Figure 1).

\section{Intestinal Barrier Function}

The integrity of the gut barrier plays a key role in prohibiting harmful intestinal materials being translocated into the bloodstream (Figure 1). All patients with various degrees of ALD have disruption of the gut barrier $(9,10)$. The oxidative and nonoxidative metabolites of ethanol produced by gut bacteria and intestinal epithelial cells disrupt the gut barrier by degrading the tight junction proteins or destroying the interaction of claudin-1 and ZO-1 (11-13). In chronic alcohol feeding mice, enteric dysbiosis-related intestinal inflammatory response causes intestinal barrier injury, and the restoration of eubiosis using non-absorbable antibiotics inhibits intestinal inflammation and barrier dysfunction (14). However, researchers find that mouse colonized with harmful C. albicans and cytolytic E. faecalis has no effect on intestinal permeability when exposed to alcohol $(6,7)$. To sum up, the relationship between intestinal dysbiosis and barrier function needs further studies.

The gut vascular barrier (GVB) is another gatekeeper that prevent bacteria translocation across the gut to reach the portal vein. Salmonella typhimurium broke the GVB in a manner that is dependent on the declined $\mathrm{Wnt} / \beta$-catenin signaling in gut vascular endothelial cells (15). In experimental cirrhosis and nonalcoholic fatty liver disease (NAFLD), farnesoid X receptor (FXR) agonists modulate the GVB to reduce bacterial translocation through driving $\beta$-catenin activation in endothelial cells $(16,17)$. Leakage of intestinal vascular endothelia has been observed in experimental ALD mice and patients with $\operatorname{ALD}(18,19)$.

\section{Gut Microbiota}

The intestinal bacteria are significantly altered in patients with ALD and experimental animals. At the phylum level, mice fed with alcohol diet have relatively higher abundances of Bacteroidetes and Verrucomicrobia when compared to mice fed with a control diet, whereas the mice fed with a control diet have a relative predominance of Firmicutes (20). However, alcoholics, with decreased communication of the microbial network in the colon (21) (Table 1), have the lower median abundances of Bacteroidetes and the higher median abundances of Proteobacteria than the healthy subjects (22). The commensal microbiota is exhausted in ALD patients with and without cirrhosis (23). At the species level, when compared with the healthy group, the numbers of Bifidobacteria, Lactobacilli, and Enterococci are significantly reduced in the alcoholics (24). Patient with alcoholic cirrhosis have 27 times more Enterobactericaea in their feces than healthy volunteers, and Enterobactericaea is the most common liver translocated bacterium in patients with cirrhosis (25).

The severity of ALD closely relates to the degree of intestinal flora alternations (26). Alcohol dependence is negatively correlated with levels of butyric-producing clostridium species (23). Severe patients with alcoholic hepatitis have increased Bacilli, Lactobacillales, Veillonella, and decreased Eubacterium_g23, Oscillibacter and Clostridiales in the fecal compared with the healthy controls (27). The reduced Akkermansia and increased Bacteroides are used to identify alcohol use disorder patients with an accuracy of 93.4\% (28). Cytolysin-positive E. faecalis is correlated with the severity of liver disease and mortality in patients with alcoholic hepatitis (34). However, a research from Arun J Sanyal el at. points out that when compared with heavy drinkers, alcoholic hepatitis have more obvious microbiome characteristics, while the bacterial signature between moderate and severe alcoholic hepatitis is not differential (35).

\section{Gut Fungi}

Except for bacteria, gut microorganisms consist of fungi, archaea, and viruses. The role of intestinal fungi in ALD has caught researchers' attention recently. Compared with the control subjects, alcoholic patients have lower fungal species richness and diversity (31). An increased systemic immune response to fungi and their products is associated with increased mortality in patients with alcoholic hepatitis (32). The overgrowth of Candida, especially C. albicans, is observed in patients with ALD compared with non-alcoholic controls, where Penicillium is dominant in the gut of non-alcoholic controls $(7,31,32)$. Moreover, the species of C. albicans are significantly decreased in alcohol abusers after 2 weeks of abstinence (33). In chronic ethanol diet feeding mice, the commensal fungus Meyerozyma guilliermondii is significantly increased compared with mice fed with control diet (36).

\section{MECHANISMS OF INTESTINAL DYSBIOSIS IN THE DEVELOPMENT OF ALD}

Pathogenic microorganism-related signals and metabolites produced by bacteria or fungi, such as short-chain fatty acids, bile acids, and $\beta$-glucan, are involved in the pathology of ALD (37). Restoring intestinal dysbiosis has been found to improve alcoholic liver injury and inflammatory response in patients and experimental mice. We will discuss the mechanism of 


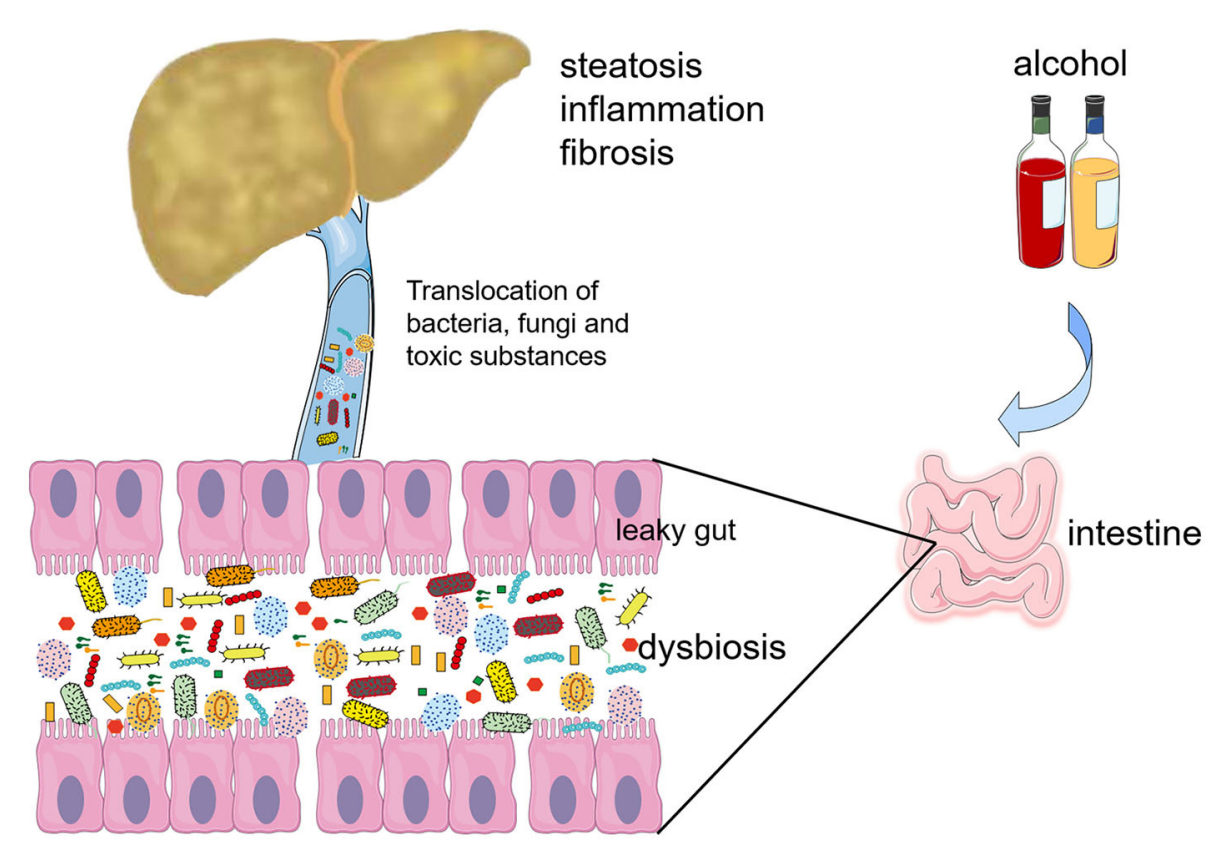

FIGURE 1 | Gut-liver cross talk in alcohol-associated liver disease (ALD). Alcohol induces the gut dysbiosis, mainly manifested as changes in the quantitative and qualitative of intestinal microbiota and fungi. The oxidative and non-oxidative metabolites of ethanol and gut dysbiosis all destroy the intestinal barrier. Gut-derived bacteria, fungi and their toxins, such as lipopolysaccharide (LPS) and $\beta$-glucan translocate into the liver though the damaged intestinal barrier and promote the progression of inflammation and fibrosis of ALD.

intestinal dysbiosis in promoting ALD development in the aspects of dysfunction of the intestinal barrier, translocated harmful materials, fatty acid metabolism immunity, bile acid homeostasis, FXR signaling, and AhR signaling (Figure 2).

\section{Dysfunction of Intestinal Barrier}

In addition to the direct effects of alcohol, several molecules have been identified as important factors that contribute greatly to alcohol-related intestinal barrier dysfunction. Hif1a knockout in mice intestinal epithelial cells results in a significantly decrease of the Firmicutes/Bacteroidetes ratio and the Lactobacillus level, exacerbating gut leakiness (38). Mucin 2, secreted by goblet cells, protects against pathogens penetrating the inner mucus layer. However, Muc2 knockout protects mice from alcoholinduced liver injury, prevents the intestinal bacterial overgrowth, and enhances the expression and activity of Reg3b and Reg3g (39). $M m p 7^{-/-}$mouse with a deficiency in active $\alpha$-defensins of intestinal paneth cells promotes PAMP translocation and worsens the liver damage of alcohol-exposed mice (40).

Immune dysfunction is another important cause of alcoholrelated intestinal barrier dysfunction. Intestinal flora is essential for maintaining the immune homeostasis of the gut-liver axis (41). On one hand, the innate and adaptive immune systems influence the component and diversity of intestinal microorganisms (42). On the other hand, gut microbiota has the potential to model intestinal immune responses in healthy and disease states (43). Alcohol feeding significantly upregulates the expression of proinflammatory cytokines interleukin-1 (IL-1) beta, tumor necrosis factor-alpha (TNF- $\alpha$ ), interleukin-6
(IL-6), monocyte chemoattractant protein-1 (MCP-1), high mobility group protein box-1 (HMGB-1), interleukin-17 (IL17), interleukin-23 (IL-23), and inducible nitric oxide synthase (iNOS) in the intestine $(44,45)$. High TNF- $\alpha$ ruptures the tight junctions by phosphorylating the myosin light chain kinase (MLCK) of intestinal epithelial cells through TNF-receptor I (14). Mice fed with a chronic ethanol diet have decreased the level of fecal immunoglobulin A (IgA) but increased the systemic level of IgA increases compared with the control mice (46). Binge-onchronic alcohol reduces the number and maturation of mucosaassociated invariant $\mathrm{T}$ cells in mice intestines (47). Gut high permeability and dysbiosis result in the highly compromised antibacterial defense of mucosa-associated invariant $\mathrm{T}$ cells in patients with ALD (48). Ethanol-induced dysbiosis lower type 3 innate lymphoid cells' production of interleukin-22 (IL22 ), and restore IL-22 produced by engineered bacteria in the intestine protecting mice from ethanol-induced steatohepatitis (49). In addition, IL-22 activates the hepatic signal transducer and activator of transcription 3 (STAT3), decreases the hepatic expression of fatty acid transport protein, and ameliorates alcohol related liver inflammatory injury and hepatic oxidative stress (50).

\section{Translocated Intestinal Bacteria and Their Products}

The translocation of intestinal bacteria and their constituent parts, such as peptidoglycan, LPS, flagellin, and CpG DNA, play an important role in ALD progression. Most studies have reported a change in LPS. LPS translocation through 
TABLE 1 | Changes in intestinal bacteria or fungi and associated metabolites in patients with alcoholic liver disease.

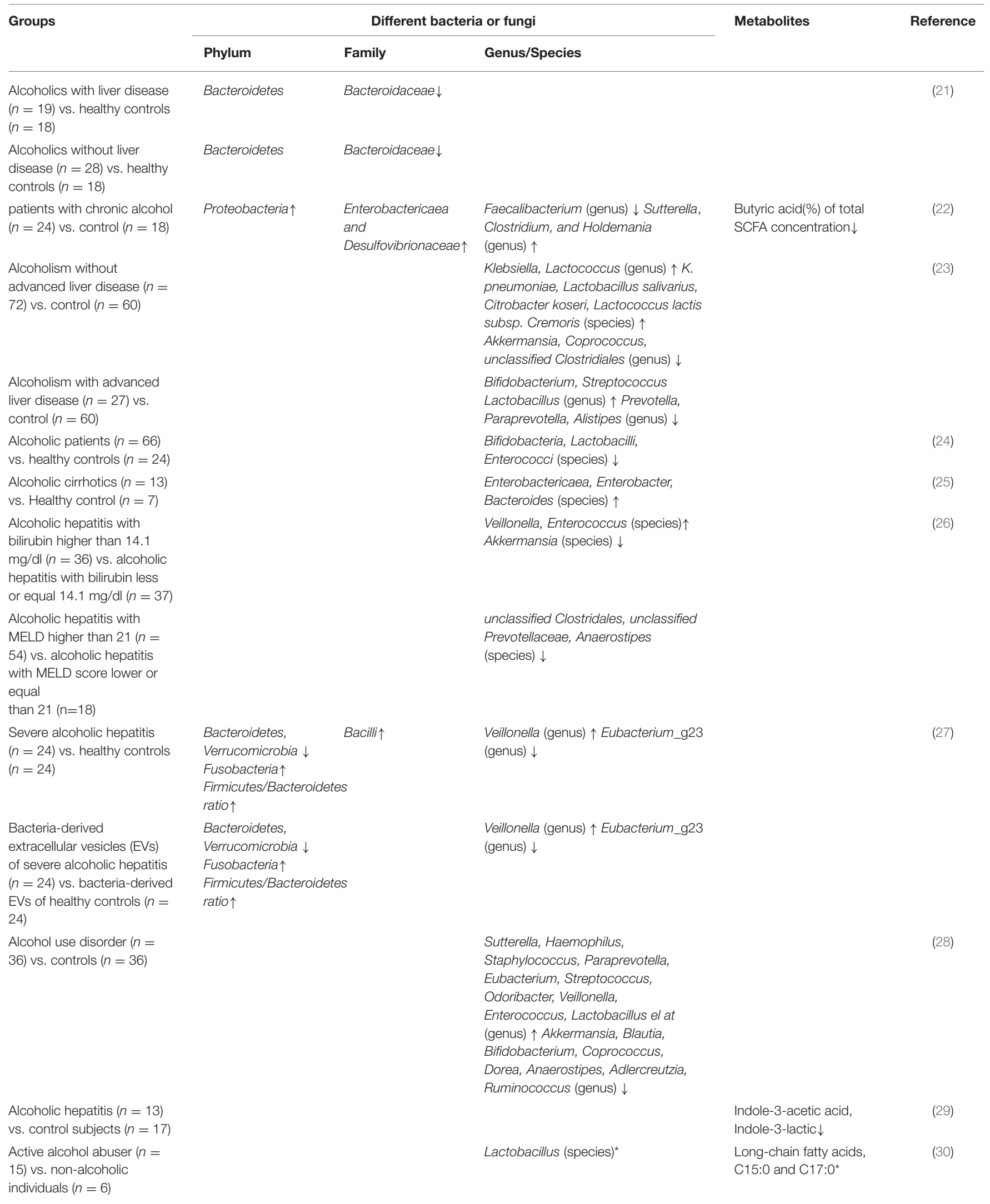


TABLE 1 | Continued

\begin{tabular}{|c|c|c|c|c|c|}
\hline Groups & \multicolumn{3}{|c|}{ Different bacteria or fungi } & Metabolites & Reference \\
\hline $\begin{array}{l}\text { Alcoholic hepatitis }(n=82) \\
\text { vs. alcohol use disorder }(n= \\
\text { 38) }\end{array}$ & & & Enterococcus faecalis $\uparrow$ & Cytolysin $\uparrow$ & \\
\hline $\begin{array}{l}\text { Alcoholic hepatitis }(n=91) \\
\text { vs. controls }(n=11)\end{array}$ & & & Candida albicans $\uparrow$ & Candidalysin $\uparrow$ & (7) \\
\hline $\begin{array}{l}\text { Alcoholic hepatitis }(n=91) \\
\text { vs. alcohol use disorders ( } n \\
=42)\end{array}$ & & & Candida albicans $\uparrow$ & Candidalysin $\uparrow$ & \\
\hline $\begin{array}{l}\text { Alcoholic hepatitis patients } \\
(n=59) \text { vs. non-alcoholic } \\
\text { controls }(n=11)\end{array}$ & & & $\begin{array}{l}\text { Candida (genus) } \uparrow \text { Penicillium, } \\
\text { Saccharomyces, Debaromyces } \\
\text { (genus) } \downarrow\end{array}$ & & \\
\hline $\begin{array}{l}\text { Alcohol use disorder ( } n= \\
66 \text { ) vs. control subjects }(n= \\
\text { 18) }\end{array}$ & & & $\begin{array}{l}\text { Candida, Debaryomyces, Pichia, } \\
\text { Kluyveromyces, Issatchenkia, } \\
\text { Scopulariopsis (genus) } \uparrow \text { C. albicans, } \\
\text { Candida zeylanoides, Issatchenkia } \\
\text { orientalis, and Scopulariopsis cordiae } \\
\text { (species) } \uparrow \text { Aspergillus (genus) } \downarrow \\
\text { Kazachstania humilis (species) } \downarrow\end{array}$ & & (33) \\
\hline
\end{tabular}

*There is significantly correlation between Lactobacilli species and levels of long-chain fatty acids, and their metabolites C15:0 and C17:0 in the fecal samples of active alcohol abusers but not in controls. $\uparrow$ means increased, $\downarrow$ means decreased.

the leaky intestine results in circulating endotoxemia and aggravates alcohol-induced liver inflammation through TLR4 signaling in liver (51). Activation of the LPS-TLR4 signaling pathway promotes the release of pro-inflammatory factors, such as TNF- $\alpha$ and IL-6. Hepatocyte TLR4 deficiency decreases lipogenic genes expression, enhances fatty acid oxidation, and reduces inflammatory genes expression of white adipose tissue, then prevents mice from alcohol-induced liver injury (29). Chronic ethanol exposure sensitizes hepatic macrophages to LPS and enhances liver inflammatory damage through TLR4 signaling (52). The absence of MyD88, one of TLR4 signaling adaptor, prevents the progression of hepatic steatosis and inflammation in chronic ethanol-exposed mice (53). However, TLR7-mediated signaling suppresses hepatic injury, steatosis, and inflammation of chronic binge ethanol fed mice (54). In addition, TLR9 signaling protects chronic alcohol exposure mice from hepatic oxidative stress but worsens hepatic inflammation.

Increased exposure to bacterial exotoxins and reduced toxin clearance in the liver also aggravate alcohol-related liver injury and inflammation. Cytolysin from E. faecalis, which caused hepatocyte death, are correlated with the severity of liver disease and mortality in patients with alcoholic hepatitis (34). The lack of $\alpha 1-2$-fucosylation in the intestine might contribute to increase the cytolytic E. faecalis in chronic ethanol exposed mice (55). Chronic ethanol feeding impairs the hepatic clearance of translocated pathobionts due to the reduced complement receptor of immunoglobulin (CRIg) expression in Kupffer cell (56).

\section{Fatty Acid Metabolism}

Fatty acid metabolism is significantly altered in ALD. Shortchain fatty acids (SCFA), the fermented product of dietary fiber, are the energy source for intestinal epithelial cells and for maintaining barrier integrity (57). The $16 \mathrm{~S}$ rRNA gene and whole genome shotgun metagenomic analysis have showed the damage of acetyl-coenzyme A (CoA) butyrate synthesizing pathway in butyrate-producing bacterial genera which caused a decreased intestinal level of butyrate by chronic ethanol feeding (58). Tributyrin can inhibit ethanol-induced intestinal barrier and liver injury $(59,60)$. Saturated long-chain fatty acids are decreased in ethanol intragastric mice, and maintaining the levels of saturated fatty acids in the intestine promote commensal Lactobacillus growth and stabilize gut barrier (30). The n3polyunsaturated fatty acids (PUFAs) can attenuate experimental ALD through decreasing neutrophil chemoattract (61).

\section{Bile Acid Homeostasis}

Bile acid homeostasis is disturbed in ALD. Gut bacteria modulate bile acid metabolism as bile acid diversity is 


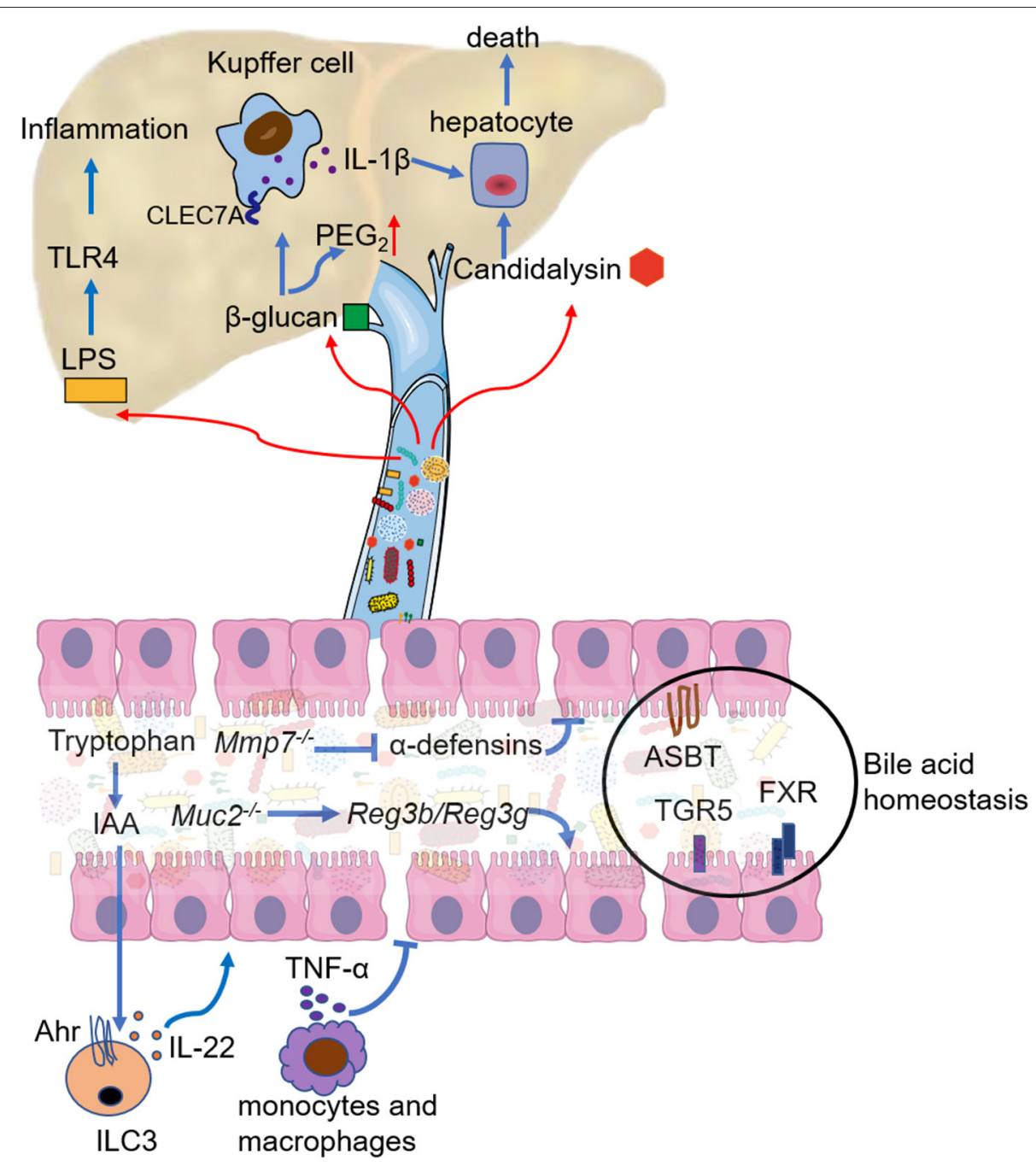

FIGURE 2 | Mechanistic contribution of the gut dysbiosis to ALD. Gut dysbiosis modulates the response of intestinal immune cells, mainly manifest as decreased IL-22 secretion by innate lymphoid cell 3 (ILC3) and increased TNF- $\alpha$ secretion by intestinal monocytes and macrophages. Both lead to the breakdown of the intestinal barrier function. Deficiency in active $\alpha$-defensins of intestinal paneth cells (Mmp7 knockout) promotes pathogen associated molecular pattern (PAMP) translocation, but mucin2 deficiency enhances the expression and activity of Reg3b and Reg3g. Bile acid homeostasis is disturbed in ALD, and the regulation of apical sodium-dependent bile salt transporter (ASBT), bile acid receptor (TGR5), and farnesoid X receptor (FXR) could restore bile acid homeostasis and ethanol-associated dysbiosis. LPS from the intestine aggravates liver inflammation mainly through TLR4 signaling. 1,3- $\beta$-glucan from the overgrowth of fungi on the one hand binds to the C-type lectin domain family 7 member A (CLEC7A) of Kupffer cells and promotes liver inflammation, on the another hand increases PGE 2 production in the liver. Candidalysin from Candida albicans has directly cytotoxic to hepatocytes.

lower and the proportion of taurine-conjugated bile acids is increased in germ-free rats compared with conventional subjects (62). Chronic alcohol administration induces the high expression of choloylglycine hydrolase that is responsible for the deconjugation of bile acid in mice bacteria and the increased levels of unconjugated bile acids, such as cholic acid and muricholic acid in the ileum and plasma, compared with control mice (63). Persons with chronic alcohol consumption have increased bile acid synthesis and bile acid pool in the liver (64). The bile-acid receptor TGR5 maintains biliary homeostasis, as TGR5 deficiency mice have intestinal microbiota dysbiosis, higher plasma and liver levels of secondary bile acids, and greater liver steatosis and inflammation when fed with ethanol diet than WT mice (65). The apical sodium-dependent bile salt transporter (ASBT) inhibitor GSK2330672 attenuates the liver injury of chronic plus binge alcohol mouse by decreasing intestinal bile acid accumulation, and increasing hepatic CYP7A1 expression (66). Restoring bile acid homeostasis by a proliferator-activated receptordelta agonist seladelpar (MBX-8025) could reduce chronic ethanol-induced liver damage and improve ethanol-associated dysbiosis (67).

\section{FXR Signaling}

The FXR plays an important role in energy metabolism and bile acid synthesis, and thus are extensively studied in ALD. 
The importance of FXR is different in the gut and the liver in ALD. Intestine-specific FXR knockout mice are more susceptible to ethanol-induced liver steatosis and inflammation compared with WT mice $(68,69)$. The intestine-restricted FXR agonist fexaramine mitigates hepatic injury and inflammation of chronic ethanol-fed mouse (63). However, FXR deletion in hepatocytes has no effect on the severity of steatosis, inflammation, or liver fibrosis in chronic plus binge alcohol feeding mice, although slight liver lipid deposition and collagen accumulation are increased (70). Nevertheless, an administration of ursodeoxycholic acid (UDCA) attenuates NF-kB activation and inflammatory infiltrations in the liver of FXR knockout mice exposed to ethanol (71). These demonstrate the significance of FXR interaction with gut microbiota in the pathophysiology of ALD.

\section{Tryptophan and AhR}

Aromatic amino acids are important factors to maintain the homeostasis of intestinal flora, among which tryptophan is fully studied in ALD. Tryptophan and tryptophan-derived metabolites are reduced in the fecal and serum of alcoholic hepatitis patients with cirrhosis when compared with nonalcoholic controls, despite that the ability of microorganisms to synthesize tryptophan has improved (72). In ALD animal experiment, Lactobacillus rhamnosus GG derived exosome enriched with bacterial metabolites of tryptophan can improve intestinal barrier function through $\mathrm{AhR}$ signaling that promotes IL22 production by intestinal immune cells (73). Alcohol-induced intestinal dysbiosis reduces intestinal IL-22 production and indoce-3-acetic acid (IAA) levels. IAA supplementation, as a microbial-derived ligand of $\mathrm{AhR}$, protects mice from ethanol-induced liver steatohepatitis by preventing bacterial translocation to liver (49). Intestinal epithelial cell-specific Ahr knockout exacerbates ethanol-induced liver injury through promoting the translocation of Helicobacter hepaticus and Helicobacter ganmani to liver (74). Ficz (6-formyllindolo (3, 2-B) carbazole), an AhR agonist, reduces ALD liver injury with similar effects to prebiotics. Moreover, alcoholfed Ahr knockout mice eliminates the beneficial effects of prebiotics (75).

\section{Gut Fungi Relevant Mechanism}

The 1,3- $\beta$-glucan from the overgrowth of fungi in chronic alcohol-fed mice binds to the C-type lectin domain family 7 member A (CLEC7A) of Kupffer cells and promotes liver inflammation (31). Patients with alcoholic hepatitis have an intense immune response to fungus, as serum anti-Saccharomyces cerevisiae antibodies are significantly high compared to patients with alcohol use disorder and nonalcoholic controls (32). Candidalysin positive C. albicans exacerbate ethanol-induced liver injury not dependent on the further impairment of intestinal barrier function through Candidalysin, but dependent on directly cytotoxic to hepatocytes (7). The commensal fungus M. guilliermondii induced alcoholic hepatic steatosis is probably due to translocated $\beta$-glucan increasing $\mathrm{PGE}_{2}$ production in the liver (36).

\section{TREATMENTS}

\section{Fecal Microbiota Transplantation}

Comparing with patients treated with the standard of care, fecal microbiota transplantation from family members attenuates the disease severity and improves the survival rate of severe patients with alcoholic hepatitis (76). In mice models, fecal microbiota transplantation from alcohol-tolerant donor mice to alcoholsensitive recipient mice can correct alcohol-induced dysbiosis and prevent alcohol-induced liver injury (77).

\section{Probiotic Bacteria}

Many probiotic therapies have been carried out in human patients with ALD and experimental ALD mice where they have received inspiring results. Patients with alcoholic hepatitis who receive 7 days of Lactobacillus subtilis/Streptococcus faecium have reduced gut-derived microbial LPS and TNF- $\alpha$ level (78). Different species of Lactobacillus, such as Lactobacillus plantarum (79), Lactobacillus acidophilus (80), Lactobacillus fermentum (81), and L. rhamnosus GG (82) are all reported to protect against alcohol-induced liver injury through improving intestinal barrier function, modulating gut bacteria, and balancing $\mathrm{T}_{\text {reg }}$ and $\mathrm{T}_{\mathrm{H}} 17$ cells in peripheral blood of mice. Akkermansia muciniphila supplementation decreases ethanolinduced gut leakiness and hepatic injury (83). L. plantarum LC27 and Bifidobacterium longum LC67 inhibit the activation of NF- $\kappa \mathrm{B}$ mediated by LPS, restore the disturbed intestinal flora, and ultimately reduce alcoholic steatosis in mice (84). Bacillus subtilis relives alcohol-induced liver damage by reducing bacterial endotoxin translocation and liver inflammation (34). A new strain of Pediococcus pentosaceus alleviates ethanolinduced liver injury by increasing the abundance of bacteria that produce SCFAs and strengthening tight junctions of intestinal epithelial cells (85). Faecalibacterium prausnitzii and potato starch supplementation attenuate chronic-binge ethanol-induced liver injury by increasing propionate abundance in mice cecum and mitigating the losses of SCFA transporter in the proximal colon (86). VSL\#3 treatment prevents intestinal bacteria and their products from spreading to portal circulation and downregulates liver inflammation mediated by TNF- $\alpha$ (87).

\section{Probiotic Fungi}

Saccharomyces cerevisiae var. boulardii which is anticarcinogenic, antibacterial antiviral, antioxidant, and able to reduce serum cholesterol level, has been used for treating various gut-related diseases (88). Saccharomyces boulardii administration attenuates acute liver injury (89). Besides, $S$. boulardii administration attenuates hepatic steatosis, lowgrade inflammation, and changes the gut microbiome (90). Hanseniaspora osmophila, Lachancea thermotolerans, and $S$. cerevisiae strains are proved to have the most potential as healthpromoting probiotics (91). However, it has also been reported that Clostridium difficile colitis and neutropenic patients have S. cerevisiae fungemia after treatment with $S$. boulardii as probiotic $(92,93)$. For future treatments, doctors should concern about the potential risk when prescribing fungal probiotics, especially to immunocompromised patients. 


\section{Prebiotics}

Some amino acids, fatty acids, and probiotic fermentation are found to alleviate alcoholic liver disease. Ethanol feeding exhausts protein thiols, raises oxidized protein thiols in mice gut, while glutamine complement can attenuate the protein thiol oxidation of distal colonic mucosa (94). What's more, glutamine prevents the ethanol-induced disruption of the tight junction by EGFR-dependent mechanism (95, 96). L-cysteine attenuates acetaldehyde-induced transepithelial electrical resistance (TEER), and inhibits the ROS injury of Caco-2 cells (11). Tributyrin supplementation has been found to attenuate both acute and chronic-binge ethanol induced intestinal leakage and liver damage $(60,97,98)$. For chronic alcohol intragastric mice, supplementation with saturated fatty acids can enhance the intestinal barrier and reduce alcoholinduced liver injury (30). The fermentation broth of the mixture of Pueraria lobata, Lonicera japonica, and Crataegus pinnatifida by L. rhamnosus 217-1 is reported to alleviate alcohol-induced intestinal microbiome disorders, and reduce oxidative stress and inflammatory signals in the liver (99).

\section{Lifestyle and Medical Intervention Diet Regulation}

Dietary inulin and flaxseed oil treatment both attenuate the hepatitis of chronic alcohol exposed mice via modulating liver inflammatory response and restoring of the gut microbiota dysbiosis (100, 101). Dietary okra seed oil consumption attenuates lipid metabolic disorder and gut dysbiosis of ALD mice (46).

\section{Traditional Medicine}

Water-insoluble polysaccharide from Wolfiporia cocos reduce liver steatosis caused by chronic ethanol feeding, and suppress the overgrowth of intestinal fungi and Proteosbacteria (36). Pomegranate prevents intestinal leakage and liver inflammatory damage caused by alcohol abuse through inhibiting the gut oxidative and nitrative stress (102). Kaempferol alleviates acute alcoholic liver injury in mice by regulating intestinal tight junction protein, butyric acid receptor, and butyric acid transporter expressions (103). Ginkgo biloba compound and puerarin ameliorate experimental alcoholic liver injury by downregulating the expressions of TNF- $\alpha$, lipopolysaccharide

\section{REFERENCES}

1. Axley PD, Richardson CT, Singal AK. Epidemiology of alcohol consumption and societal burden of alcoholism and alcoholic liver disease. Clin Liver Dis. (2019) 23:39-50. doi: 10.1016/j.cld.2018.09.011

2. Seitz HK, Bataller R, Cortez-Pinto H, Gao B, Gual A, Lackner C, et al. Alcoholic liver disease. Nat Rev Dis Primers. (2018) 4:16. doi: 10.1038/s41572-018-0014-7

3. Llopis M, Cassard AM, Wrzosek L, Boschat L, Bruneau A, Ferrere G, et al. Intestinal microbiota contributes to individual susceptibility to alcoholic liver disease. Gut. (2016) 65:830-9. doi: 10.1136/gutjnl-2015-310585

4. Louvet A, Mathurin P. Alcoholic liver disease: mechanisms of injury and targeted treatment. Nat Rev Gastroenterol Hepatol. (2015) 12:23142. doi: 10.1038/nrgastro.2015.35 binding protein (LBP), CD14, and TLR4 in liver, and upregulating the expression of tight junction proteins in the intestine $(104,105)$. The rice bran phenolic extract relieves alcohol caused intestinal microbiota dysbiosis, barrier dysfunction, and liver inflammation (106).

\section{CONCLUSIONS}

Gut dysbiosis promotes the development of ALD. The role of fungi in ALD is also important, which deserves further study. As an important barrier for pathogenic microorganisms from the intestine to the portal vein, the mechanism of intestinal blood barrier injury in ALD needs to be clarified. A research recently pointed out chronic alcohol exposure will result in insufficient anti-bacterial immunity of the body, as the number of MAIT cells in peripheral blood of patients with alcohol-related cirrhosis was significantly reduced and their function was impaired (48). Attention should be paid to the high risk of bacteria infection in ALD patients. There is a clear causal link between intestinal translocation PAMPs and liver inflammation (40). Therefore, it is of great significance to further elucidate the mechanism of intestinal barrier injury in ALD. Microecological disorder is an important cause of intestinal barrier impairment, and microbiota-based treatments are the powerful therapeutic options for ALD (107).

\section{AUTHOR CONTRIBUTIONS}

HC, LY, and XH designed the review and revised the manuscript. LC collected the data and drafted the manuscript. YZ revised the manuscript. All authors have approved the final version.

\section{FUNDING}

This study was supported by the National Natural Science Foundation of China (No. 82000561 to HC; Nos. 81974078, 81570530, and 81370550 to LY; Nos. 81974062 and 81720108006 to XH), Department of Science and Technology, Hubei Provincial People's Government (No. 2020FCA014 to XH), and the Science foundation of union hospital (No. 2021xhyn005 to $\mathrm{HC})$.
5. Bajaj J S. Alcohol, liver disease and the gut microbiota. Nat Rev Gastroenterol Hepatol. (2019) 16:235-46. doi: 10.1038/s41575-018-0099-1

6. Duan Y, Llorente C, Lang S, Brandl K, Chu H, Jiang L, et al. Bacteriophage targeting of gut bacterium attenuates alcoholic liver disease. Nature. (2019) 575:505-11. doi: 10.1038/s41586-019-1742-X

7. Chu H, Duan Y, Lang S, Jiang L, Wang Y, Llorente C, et al. The Candida albicans exotoxin candidalysin promotes alcohol-associated liver disease. J Hepatol. (2020) 72:391-400. doi: 10.1016/j.jhep.2019. 09.029

8. Hartmann P, Seebauer C T, Schnabl B. Alcoholic liver disease: the gut microbiome and liver cross talk. Alcohol Clin Exp Res. (2015) 39:76375. doi: 10.1111/acer.12704

9. Urbaschek R, Mccuskey R S, Rudi V, Becker K P, Stickel F, Urbaschek B, et al. Endotoxin, endotoxin-neutralizing-capacity, sCD14, sICAM-1, and 
cytokines in patients with various degrees of alcoholic liver disease. Alcohol Clin Exp Res. (2001) 25:261-8. doi: 10.1111/j.1530-0277.2001.tb02207.x

10. Fukui H. Relation of endotoxin, endotoxin binding proteins and macrophages to severe alcoholic liver injury and multiple organ failure. Alcohol Clin Exp Res. (2005) 29 (11 Suppl):172S-9S. doi: 10.1097/01.alc.0000189278.30237.e9

11. Elamin E, Masclee A, Troost F, Dekker J, Jonkers D. Activation of the epithelial-to-mesenchymal transition factor snail mediates acetaldehydeinduced intestinal epithelial barrier disruption. Alcohol Clin Exp Res. (2014) 38:344-53. doi: 10.1111/acer.12234

12. Pannequin J, Delaunay N, Darido C, Maurice T, Crespy P, Frohman MA, et al. Phosphatidylethanol accumulation promotes intestinal hyperplasia by inducing ZONAB-mediated cell density increase in response to chronic ethanol exposure. Mol Cancer Res. (2007) 5:1147-57. doi: 10.1158/1541-7786.MCR-07-0198

13. Cho YE, Yu LR, Abdelmegeed MA, Yoo SH, Song J. Apoptosis of enterocytes and nitration of junctional complex proteins promote alcoholinduced gut leakiness and liver injury. J Hepatol. (2018) 69:14253. doi: 10.1016/j.jhep.2018.02.005

14. Chen P, Stärkel P, Turner JR, Ho SB, Schnabl B. Dysbiosis-induced intestinal inflammation activates tumor necrosis factor receptor I and mediates alcoholic liver disease in mice. Hepatology. (2015) 61:88394. doi: 10.1002/hep. 27489

15. Bouziat R, Jabri B. IMMUNOLOGY. Breaching the gut-vascular barrier. Science. (2015) 350:742-3. doi: 10.1126/science.aad6768

16. Sorribas M, Jakob MO, Yilmaz B, Li H, Stutz D, Noser Y, et al. FXR modulates the gut-vascular barrier by regulating the entry sites for bacterial translocation in experimental cirrhosis. J Hepatol. (2019) 71:112640. doi: 10.1016/j.jhep.2019.06.017

17. Mouries J, Brescia P, Silvestri A, Spadoni I, Sorribas M, Wiest R, et al. Microbiota-driven gut vascular barrier disruption is a prerequisite for non-alcoholic steatohepatitis development. J Hepatol. (2019) 71:121628. doi: 10.1016/j.jhep.2019.08.005

18. Maccioni L, Gao B, Leclercq S, Pirlot B, Horsmans Y, De Timary $\mathrm{P}$, et al. Intestinal permeability, microbial translocation, changes in duodenal and fecal microbiota, and their associations with alcoholic liver disease progression in humans. Gut Microbes. (2020) 12:1782157. doi: 10.1080/19490976.2020.1782157

19. Grander C, Grabherr F, Spadoni I, Enrich B, Oberhuber G, Rescigno $\mathrm{M}$, et al. The role of gut vascular barrier in experimental alcoholic liver disease and A. muciniphila supplementation. Gut Microbes. (2020) 12:1851986. doi: 10.1080/19490976.2020.1851986

20. Yan AW, Fouts DE, Brandl J, Stärkel P, Torralba M, Schott E, et al. Enteric dysbiosis associated with a mouse model of alcoholic liver disease. Hepatology. (2011) 53:96-105. doi: 10.1002/hep.24018

21. Mutlu EA, Gillevet PM, Rangwala H, Sikaroodi M, Naqvi A, Engen PA, et al. Colonic microbiome is altered in alcoholism. Am J Physiol Gastrointest Liver Physiol. (2012) 302:G966-78. doi: 10.1152/ajpgi.00380.2011

22. Bjørkhaug ST, Aanes H, Neupane SP, Bramness JG, Malvik S, Henriksen $\mathrm{C}$, et al. Characterization of gut microbiota composition and functions in patients with chronic alcohol overconsumption. Gut Microbes. (2019) 10:663-75. doi: 10.1080/19490976.2019.1580097

23. Dubinkina VB, Tyakht AV, Odintsova VY, Yarygin KS, Kovarsky BA, Pavlenko AV, et al. Links of gut microbiota composition with alcohol dependence syndrome and alcoholic liver disease. Microbiome. (2017) 5:141. doi: 10.1186/s40168-017-0359-2

24. Kirpich IA, Solovieva NV, Leikhter SN, Shidakova NA, Lebedeva OV, Sidorov PI, et al. Probiotics restore bowel flora and improve liver enzymes in human alcohol-induced liver injury: a pilot study. Alcohol. (2008) 42:67582. doi: 10.1016/j.alcohol.2008.08.006

25. Tuomisto S, Pessi T, Collin P, Vuento R, Aittoniemi J, Karhunen PJ. Changes in gut bacterial populations and their translocation into liver and ascites in alcoholic liver cirrhotics. BMC Gastroenterol. (2014) 14:40. doi: 10.1186/1471-230X-14-40

26. Lang S, Fairfied B, Gao B, Duan Y, Zhang X, Fouts DE, et al. Changes in the fecal bacterial microbiota associated with disease severity in alcoholic hepatitis patients. Gut Microbes. (2020) 12:1785251. doi: $10.1080 / 19490976.2020 .1785251$
27. Kim SS, Eun JW, Cho HJ, Song DS, Kim CW, Kim YS, et al. Microbiome as a potential diagnostic and predictive biomarker in severe alcoholic hepatitis. Aliment Pharmacol Ther. (2021) 53:540-51. doi: 10.1111/apt.16200

28. Addolorato G, Ponziani FR, Dionisi T, Mosoni C, Vassallo GA, Sestito L, et al. Gut microbiota compositional and functional fingerprint in patients with alcohol use disorder and alcohol-associated liver disease. Liver Int. (2020) 40:878-88. doi: 10.1111/liv.14383

29. Jia L, Chang X, Qian S, Liu C, Lord CC, Ahmed N, et al. Hepatocyte toll-like receptor 4 deficiency protects against alcohol-induced fatty liver disease. Mol Metab. (2018) 14:121-9. doi: 10.1016/j.molmet.2018.05.015

30. Chen P, Torralba M, Tan J, Embree M, Zengler K, Stärkel P, et al. Supplementation of saturated long-chain fatty acids maintains intestinal eubiosis and reduces ethanol-induced liver injury in mice. Gastroenterology. (2015) 148:203-14.e16. doi: 10.1053/j.gastro.2014.09.014

31. Yang AM, Inamine $\mathrm{T}$, Hochrath $\mathrm{K}$, Chen $\mathrm{P}$, Wang L, Llorente $\mathrm{C}$, et al. Intestinal fungi contribute to development of alcoholic liver disease. J Clin Invest. (2017) 127:2829-41. doi: 10.1172/JCI90562

32. Lang S, Duan Y, Liu J, Torralba MG, Kuelbs C, Ventura-Cots M, et al. Intestinal fungal dysbiosis and systemic immune response to fungi in patients with alcoholic hepatitis. Hepatology. (2020) 71:52238. doi: 10.1002/hep.30832

33. Hartmann P, Lang S, Zeng S, Duan Y, Zhang X, Wang Y, et al. Dynamic changes of the fungal microbiome in alcohol use disorder. Front Physiol. (2021) 12:699253. doi: 10.3389/fphys.2021.699253

34. Gao B, Emami A, Nath S, Schnabl B. Microbial products and metabolites contributing to alcohol-related liver disease. Mol Nutr Food Res. (2021) 65:e2000023. doi: 10.1002/mnfr.202000023

35. Smirnova E, Puri P, Muthiah MD, Daitya K, Brown R, Chalasani N, et al. Fecal microbiome distinguishes alcohol consumption from alcoholic hepatitis but does not discriminate disease severity. Hepatology. (2020) 72:271-86. doi: 10.1002/hep.31178

36. Sun S, Wang K, Sun L, Cheng B, Qiao S, Dai H, et al. Therapeutic manipulation of gut microbiota by polysaccharides of reveals the contribution of the gut fungi-induced PGE to alcoholic hepatic steatosis. Gut Microbes. (2020) 12:1830693. doi: 10.1080/19490976.2020.1830693

37. Albillos A, De Gottardi A, Rescigno M. The gut-liver axis in liver disease: Pathophysiological basis for therapy. J Hepatol. (2020) 72:55877. doi: 10.1016/j.jhep.2019.10.003

38. Shao T, Zhao C, Li F, Gu Z, Liu L, Zhang L, et al. Intestinal HIF- $1 \alpha$ deletion exacerbates alcoholic liver disease by inducing intestinal dysbiosis and barrier dysfunction. J Hepatol. (2018) 69:886-95. doi: 10.1016/j.jhep.2018.05.021

39. Hartmann P, Chen P, Wang HJ, Wang L, Mccole DF, Brandl K, et al. Deficiency of intestinal mucin-2 ameliorates experimental alcoholic liver disease in mice. Hepatology. (2013) 58:108-19. doi: 10.1002/hep.26321

40. Zhong W, Wei X, Hao L, Lin TD, Yue R, Sun X, et al. Paneth cell dysfunction mediates alcohol-related steatohepatitis through promoting bacterial translocation in mice: role of zinc deficiency. Hepatology. (2020) 71:1575-91. doi: 10.1002/hep.30945

41. Yang X, Lu D, Zhuo J, Lin Z, Yang M, Xu X. The gut-liver axis in immune remodeling: new insight into liver diseases. Int J Biol Sci. (2020) 16:235766. doi: 10.7150/ijbs.46405

42. Konturek PC, Harsch IA, Konturek K, Schink M, Konturek T, Neurath MF, et al. Gut?Liver Axis: How Do Gut Bacteria Influence the Liver? Med Sci (Basel). (2018) 6:79. doi: 10.3390/medsci6030079

43. Round JL, Mazmanian SK. The gut microbiota shapes intestinal immune responses during health and disease. Nat Rev Immunol. (2009) 9:31323. doi: $10.1038 /$ nri2515

44. Lowe P P, Gyongyosi B, Satishchandran A, Iracheta-Vellve A, Cho Y, Ambade A, et al. Reduced gut microbiome protects from alcohol-induced neuroinflammation and alters intestinal and brain inflammasome expression. J Neuroinflammation. (2018) 15:298. doi: 10.1186/s12974-018-1328-9

45. Fleming S, Toratani S, Shea-Donohue T, Kashiwabara Y, Vogel SN, Metcalf ES. Pro- and anti-inflammatory gene expression in the murine small intestine and liver after chronic exposure to alcohol. Alcohol Clin Exp Res. (2001) 25:579-89. doi: 10.1111/j.1530-0277.2001.tb 02253.x 
46. Inamine $\mathrm{T}$, Yang $\mathrm{AM}$, Wang $\mathrm{L}$, Lee KC, Llorente $\mathrm{C}$, Schnabl B. Genetic loss of immunoglobulin a does not influence development of alcoholic steatohepatitis in mice. Alcohol Clin Exp Res. (2016) 40:260413. doi: 10.1111/acer.13239

47. Gu M, Samuelson DR, Taylor CM, Molina PE, Luo M, Siggins RW, et al. Alcohol-associated intestinal dysbiosis alters mucosal-associated invariant T-cell phenotype and function. Alcohol Clin Exp Res. (2021) 45:93447. doi: 10.1111/acer.14589

48. Riva A, Patel V, Kurioka A, Jeffery HC, Wright G, Tarff S, et al. Mucosa-associated invariant $\mathrm{T}$ cells link intestinal immunity with antibacterial immune defects in alcoholic liver disease. Gut. (2018) 67:91830. doi: 10.1136/gutjnl-2017-314458

49. Hendrikx T, Duan Y, Wang Y, Oh JH, Alexander LM, Huang W, et al. Bacteria engineered to produce IL-22 in intestine induce expression of REG3G to reduce ethanol-induced liver disease in mice. Gut. (2019) 68:1504-15. doi: 10.1136/gutjnl-2018-317232

50. Ki SH, Park O, Zheng M, Morales-Ibanez O, Kolls JK, Bataller $\mathrm{R}$, et al. Interleukin-22 treatment ameliorates alcoholic liver injury in a murine model of chronic-binge ethanol feeding: role of signal transducer and activator of transcription 3. Hepatology. (2010) 52:1291300. doi: 10.1002/hep.23837

51. An L, Wirth U, Koch D, Schirren M, Drefs M, Koliogiannis D, et al. The role of gut-derived lipopolysaccharides and the intestinal barrier in fatty liver diseases. J Gastrointest Surg. (2021). doi: 10.1007/s11605-021-05188-7. [Epub ahead of print].

52. Saikia P, Roychowdhury S, Bellos D, Pollard KA, Mcmullen MR, Mccullough RL, et al. Hyaluronic acid 35 normalizes TLR4 signaling in Kupffer cells from ethanol-fed rats via regulation of microRNA291b and its target Tollip. Sci Rep. (2017) 7:15671. doi: 10.1038/s41598-017-15760-4

53. Zhou H, Yu M, Roychowdhury S, Sanz-Garcia C, Pollard KA, Mcmullen MR, et al. Myeloid-MyD88 Contributes to Ethanol-Induced Liver Injury in Mice Linking Hepatocellular Death to Inflammation. Alcohol Clin Exp Res. (2017) 41:719-26. doi: 10.1111/acer.13345

54. Wang Q, Kim SY, Matsushita H, Wang Z, Pandyarajan V, Matsuda M, et al. Oral administration of PEGylated TLR7 ligand ameliorates alcoholassociated liver disease via the induction of IL-22. Proc Natl Acad Sci U S A. (2021) 118:e2020868118. doi: 10.1073/pnas.2020868118

55. Zhou R, Llorente C, Cao J, Gao B, Duan Y, Jiang L, et al. Deficiency of Intestinal $\alpha 1-2$-Fucosylation Exacerbates Ethanol-Induced Liver Disease in Mice. Alcohol Clin Exp Res. (2020) 44:1842-51. doi: 10.1111/acer.14405

56. Duan Y, Chu H, Brandl K, Jiang L, Zeng S, Meshgin N, et al. CRIg on liver macrophages clears pathobionts and protects against alcoholic liver disease. Nat Commun. (2021) 12:7172. doi: 10.1038/s41467-02127385-3

57. Fairfield B, Schnabl B. Gut dysbiosis as a driver in alcohol-induced liver injury. JHEP Rep. (2021) 3:100220. doi: 10.1016/j.jhepr.2020.100220

58. Singhal R, Donde H, Ghare S, Stocke K, Zhang J, Vadhanam M, et al. Decrease in acetyl-CoA pathway utilizing butyrate-producing bacteria is a key pathogenic feature of alcohol-induced functional gut microbial dysbiosis and development of liver disease in mice. Gut Microbes. (2021) 13:1946367. doi: 10.1080/19490976.2021.1946367

59. Donde H, Ghare S, Joshi-Barve S, Zhang J, Vadhanam MV, Gobejishvili L, et al. Tributyrin Inhibits Ethanol-Induced Epigenetic Repression of CPT-1A and Attenuates Hepatic Steatosis and Injury. Cell Mol Gastroenterol Hepatol. (2020) 9:569-85. doi: 10.1016/j.jcmgh.2019.10.005

60. Cresci GA, Glueck B, Mcmullen R, Xin W, Allende D, Nagy LE. Prophylactic tributyrin treatment mitigates chronic-binge ethanol-induced intestinal barrier and liver injury. J Gastroenterol Hepatol. (2017) 32:158797. doi: 10.1111 /jgh.13731

61. Warner J, Hardesty J, Song Y, Sun R, Deng Z, Xu R, et al. Transgenic mice with augmented $\mathrm{n} 3$-Polyunsaturated fatty acids are protected from liver injury caused by acute-on-chronic ethanol administration. Front Pharmacol. (2021) 12:711590. doi: 10.3389/fphar.2021.711590

62. Swann JR, Want EJ, Geier FM, Spagou K, Wilson ID, Sidaway JE, et al. Systemic gut microbial modulation of bile acid metabolism in host tissue compartments. Proc Natl Acad Sci U S A. (2011) 108 Suppl 1(Suppl 1):452330. doi: $10.1073 /$ pnas. 1006734107
63. Hartmann P, Hochrath K, Horvath A, Chen P, Seebauer CT, Llorente C, et al. Modulation of the intestinal bile acid/farnesoid X receptor/fibroblast growth factor 15 axis improves alcoholic liver disease in mice. Hepatology. (2018) 67:2150-66. doi: 10.1002/hep.29676

64. Manley S, Ding W. Role of farnesoid X receptor and bile acids in alcoholic liver disease. Acta Pharm Sin B. (2015) 5:158-67. doi: 10.1016/j.apsb.2014.12.011

65. Spatz M, Ciocan D, Merlen G, Rainteau D, Humbert L, Gomes-Rochette N, et al. Bile acid-receptor TGR5 deficiency worsens liver injury in alcoholfed mice by inducing intestinal microbiota dysbiosis. JHEP Rep. (2021) 3:100230. doi: 10.1016/j.jhepr.2021.100230

66. Matye DJ, Li Y, Chen C, Chao X, Wang H, Ni H, et al. Gut-restricted apical sodium-dependent bile acid transporter inhibitor attenuates alcoholinduced liver steatosis and injury in mice. Alcohol Clin Exp Res. (2021) 45:1188-99. doi: 10.1111/acer.14619

67. Chu H, Jiang L, Gao B, Gautam N, Alamoudi JA, Lang S, et al. The selective PPAR-delta agonist seladelpar reduces ethanol-induced liver disease by restoring gut barrier function and bile acid homeostasis in mice. Transl Res. (2021) 227:1-14. doi: 10.1016/j.trsl.2020.06.006

68. Huang M, Kong B, Zhang M, Rizzolo D, Armstrong LE, Schumacher JD, et al. Enhanced alcoholic liver disease in mice with intestinespecific farnesoid X receptor deficiency. Lab Invest. (2020) 100:115868. doi: 10.1038/s41374-020-0439-y

69. Kong B, Zhang M, Huang M, Rizzolo D, Armstrong LE, Schumacher JD, et al. FXR deficiency alters bile acid pool composition and exacerbates chronic alcohol induced liver injury. Dig Liver Dis. (2019) 51:5706. doi: 10.1016/j.dld.2018.12.026

70. Zhang M, Kong B, Huang M, Wan R, Armstrong LE, Schumacher JD, et al. FXR deletion in hepatocytes does not affect the severity of alcoholic liver disease in mice. Dig Liver Dis. (2018) 50:106875. doi: 10.1016/j.dld.2018.04.009

71. Wu WB, Chen YY, Zhu B, Peng XM, Zhang SW, Zhou ML. Excessive bile acid activated NF-kappa B and promoted the development of alcoholic steatohepatitis in farnesoid X receptor deficient mice. Biochimie. (2015) 115:86-92. doi: 10.1016/j.biochi.2015.05.014

72. Gao B, Duan Y, Lang S, Barupal D, Wu TC, Valdiviez L, et al. Functional microbiomics reveals alterations of the gut microbiome and host cometabolism in patients with alcoholic hepatitis. Hepatol Commun. (2020) 4:1168-82. doi: 10.1002/hep4.1537

73. Gu Z, Li F, Liu Y, Jiang M, Zhang L, He L, et al. Exosome-like nanoparticles from GG protect against alcohol-associated liver disease through intestinal aryl hydrocarbon receptor in mice. Hepatol Commun. (2021) 5:84664. doi: 10.1002/hep4.1679

74. Qian M, Liu J, Zhao D, Cai P, Pan C, Jia W, et al. Aryl hydrocarbon receptor deficiency in intestinal epithelial cells aggravates alcoholrelated liver disease. Cell Mol Gastroenterol Hepatol. (2022) 1:233-56. doi: 10.1016/j.jcmgh.2021.08.014

75. Wrzosek L, Ciocan D, Hugot C, Spatz M, Dupeux M, Houron C, et al. Microbiota tryptophan metabolism induces aryl hydrocarbon receptor activation and improves alcohol-induced liver injury. Gut. (2021) 70:1299308. doi: 10.1136/gutjnl-2020-321565

76. Philips CA, Pande A, Shasthry SM, Jamwal KD, Khillan V, Chandel SS, et al. Healthy donor fecal microbiota transplantation in steroid-ineligible severe alcoholic hepatitis: a pilot study. Clin Gastroenterol Hepatol. (2017) 15:600-2. doi: 10.1016/j.cgh.2016.10.029

77. Ferrere G, Wrzosek L, Cailleux F, Turpin W, Puchois V, Spatz M, et al. Fecal microbiota manipulation prevents dysbiosis and alcohol-induced liver injury in mice. J Hepatol. (2017) 66:806-15. doi: 10.1016/j.jhep.2016.11.008

78. Han SH, Suk KT, Kim DJ, Kim MY, Baik SK, Kim YD, et al. Effects of probiotics (cultured Lactobacillus subtilis/Streptococcus faecium) in the treatment of alcoholic hepatitis: randomizedcontrolled multicenter study. Eur J Gastroenterol Hepatol. (2015) 27:1300-6. doi: 10.1097/MEG.0000000000000458

79. Fang TJ, Guo JT, Lin MK, Lee MS, Chen YL, Lin WH. Protective effects of Lactobacillus plantarum against chronic alcohol-induced liver injury in the murine model. Appl Microbiol Biotechnol. (2019) 103:8597608. doi: $10.1007 /$ s00253-019-10122-8 
80. Li H, Shi J, Zhao L, Guan J, Liu F, Huo G, et al. KLDS1.0344 and KLDS1.0901 Mixture prevents chronic alcoholic liver injury in mice by protecting the intestinal barrier and regulating gut microbiota and liver-related pathways. $J$ Agric Food Chem. (2021) 69:183-97. doi: 10.1021/acs.jafc.0c06346

81. Kim BK, Lee IO, Tan PL, Eor JY, Hwang JK, Kim SH. Protective Effect of LA12 in an Alcohol-Induced Rat Model of Alcoholic Steatohepatitis. Korean J Food Sci Anim Resour. (2017) 37:931-9. doi: 10.1016/j.toxlet.2015.06.006

82. Chen RC, Xu LM, Du SJ, Huang SS, Wu H, Dong JJ, et al. Lactobacillus rhamnosus GG supernatant promotes intestinal barrier function, balances Treg and TH17 cells and ameliorates hepatic injury in a mouse model of chronic-binge alcohol feeding. Toxicol Lett. (2016) 241:10310. doi: 10.1016/j.toxlet.2015.11.019

83. Grander C, Adolph TE, Wieser V, Lowe P, Wrzosek L, Gyongyosi $B$, et al. Recovery of ethanol-induced Akkermansia muciniphila depletion ameliorates alcoholic liver disease. Gut. (2018) 67:891-901. doi: 10.1136/gutjnl-2016-313432

84. Kim WG, Kim HI, Kwon EK, Han MJ, Kim DH. Lactobacillus plantarum LC27 and Bifidobacterium longum LC67 mitigate alcoholic steatosis in mice by inhibiting LPS-mediated NF- $\kappa$ B activation through restoration of the disturbed gut microbiota. Food Funct. (2018) 9:425565. doi: 10.1039/C8FO00252E

85. Jiang XW, Li YT, Ye JZ, Lv LX, Yang LY, Bian XY, et al. New strain of alleviates ethanol-induced liver injury by modulating the gut microbiota and short-chain fatty acid metabolism. World J Gastroenterol. (2020) 26:622440. doi: 10.3748/wjg.v26.i40.6224

86. Roychowdhury S, Glueck B, Han Y, Mohammad MA, Cresci GAM. A designer synbiotic attenuates chronic-binge ethanol-induced gut-liver injury in mice. Nutrients. (2019) 11:97. doi: 10.3390/nu11010097

87. Chang B, Sang L, Wang Y, Tong J, Zhang D, Wang B. The protective effect of VSL\#3 on intestinal permeability in a rat model of alcoholic intestinal injury. BMC Gastroenterol. (2013) 13:151. doi: 10.1186/1471-230X-13-151

88. Ansari F, Alian Samakkhah S, Bahadori A, Jafari SM, Ziaee M, Khodayari MT, et al. Health-promoting properties of as a probiotic; characteristics, isolation, and applications in dairy products. Crit Rev Food Sci Nutr. (2021). doi: 10.1080/10408398.2021.1949577. [Epub ahead of print].

89. Yu L, Zhao XK, Cheng ML, Yang GZ, Wang B, Liu HJ, et al. Saccharomyces boulardii Administration Changes Gut Microbiota and Attenuates D-Galactosamine-Induced Liver Injury. Sci Rep. (2017) 7:1359. doi: 10.1038/s41598-017-01271-9

90. Everard A, Matamoros S, Geurts L, Delzenne NM, Cani PD. Saccharomyces boulardii administration changes gut microbiota and reduces hepatic steatosis, low-grade inflammation, and fat mass in obese and type 2 diabetic db/db mice. MBio. (2014) 5:e01011-4. doi: 10.1128/mBio.01011-14

91. Fernández-Pacheco P, Pintado C, Briones Pérez A, Arévalo-Villena M. Potential Probiotic Strains of and Non-: Functional and Biotechnological Characteristics. J Fungi (Basel). (2021) 7:177. doi: 10.3390/jof7030177

92. Santino I, Alari A, Bono S, Teti E, Marangi M, Bernardini A, et al. Saccharomyces cerevisiae fungemia, a possible consequence of the treatment of Clostridium difficile colitis with a probioticum. Int J Immunopathol Pharmacol. (2014) 27:143-6. doi: 10.1177/039463201402700120

93. Cesaro S, Chinello P, Rossi L, Zanesco L. Saccharomyces cerevisiae fungemia in a neutropenic patient treated with Saccharomyces boulardii. Support Care Cancer. (2000) 8:504-5. doi: 10.1007/s005200000123

94. Chaudhry KK, Shukla PK, Mir H, Manda B, Gangwar R, Yadav N, et al. Glutamine supplementation attenuates ethanol-induced disruption of apical junctional complexes in colonic epithelium and ameliorates gut barrier dysfunction and fatty liver in mice. J Nutr Biochem. (2016) 27:1626. doi: 10.1016/j.jnutbio.2015.08.012

95. Meena AS, Shukla PK, Sheth P, Rao R. EGF receptor plays a role in the mechanism of glutamine-mediated prevention of alcohol-induced gut barrier dysfunction and liver injury. J Nutr Biochem. (2019) 64:128143. doi: 10.1016/j.jnutbio.2018.10.016

96. Seth A, Basuroy S, Sheth P, Rao RK. L-Glutamine ameliorates acetaldehyde-induced increase in paracellular permeability in Caco-2 cell monolayer. Am J Physiol Gastrointest Liver Physiol. (2004) 287:G510-7. doi: 10.1152/ajpgi.00058.2004

97. Glueck B, Han Y, Cresci GAM. Tributyrin supplementation protects immune responses and vasculature and reduces oxidative stress in the proximal colon of mice exposed to chronic-binge ethanol feeding. J Immunol Res. (2018) 2018:9671919. doi: 10.1155/2018/9671919

98. Cresci GA, Bush K, Nagy LE. Tributyrin supplementation protects mice from acute ethanol-induced gut injury. Alcohol Clin Exp Res. (2014) 38:1489501. doi: 10.1111/acer.12428

99. Wang T, Wang Z, Yang Z, Cui X, Yan L, Xu Z, et al. Effect of the Fermentation Broth of the Mixture of Pueraria lobata, Lonicera japonica, and Crataegus pinnatifida by Lactobacillus rhamnosus and by 217-1 on Liver Health and Intestinal Flora in Mice With Alcoholic Liver Disease Induced by Liquor. Front Microbiol. (2021) 12:722171. doi: 10.3389/fmicb.2021. 722171

100. Yang $\mathrm{X}, \mathrm{He} \mathrm{F}$, Zhang $\mathrm{Y}$, Xue J, Li K, Zhang $\mathrm{X}$, et al. Inulin ameliorates alcoholic liver disease via suppressing LPS-TLR4-M $\psi$ axis and modulating gut microbiota in mice. Alcohol Clin Exp Res. (2019) 43:41124. doi: 10.1111/acer.13950

101. Zhang X, Wang H, Yin P, Fan H, Sun L, Liu Y. Flaxseed oil ameliorates alcoholic liver disease via anti-inflammation and modulating gut microbiota in mice. Lipids Health Dis. (2017) 16:44. doi: 10.1186/s12944-0170431-8

102. Cho YE, Song BJ. Pomegranate prevents binge alcohol-induced gut leakiness and hepatic inflammation by suppressing oxidative and nitrative stress. Redox Biol. (2018) 18:266-78. doi: 10.1016/j.redox.2018.07.012

103. Chen J, Xuan YH, Luo MX, Ni XG, Ling LQ, Hu SJ, et al. Kaempferol alleviates acute alcoholic liver injury in mice by regulating intestinal tight junction proteins and butyrate receptors and transporters. Toxicology. (2020) 429:152338. doi: 10.1016/j.tox.2019.152338

104. Li H, Qiu P, Wang J, Niu C, Pan S. Effects of compound Ginkgo biloba on intestinal permeability in rats with alcohol-induced liver injury. Food Funct. (2015) 6:470-8. doi: 10.1039/C4FO00739E

105. Peng JH, Cui T, Huang F, Chen L, Zhao Y, Xu L, et al. Puerarin ameliorates experimental alcoholic liver injury by inhibition of endotoxin gut leakage, Kupffer cell activation, and endotoxin receptors expression. J Pharmacol Exp Ther. (2013) 344:646-54. doi: 10.1124/jpet.112.201137

106. Xiao J, Zhang R, Wu Y, Wu C, Jia X, Dong L, et al. Rice bran phenolic extract protects against alcoholic liver injury in mice by alleviating intestinal microbiota dysbiosis, barrier dysfunction, and liver inflammation mediated by the endotoxin-TLR4-NF-кB pathway. J Agric Food Chem. (2020) 68:123747. doi: 10.1021/acs.jafc.0c04539

107. Sung H, Kim S W, Hong M, Suk K T. Microbiota-based treatments in alcoholic liver disease. World J Gastroenterol. (2016) 22:667382. doi: $10.3748 /$ wjg.v22.i29.6673

Conflict of Interest: The authors declare that the research was conducted in the absence of any commercial or financial relationships that could be construed as a potential conflict of interest.

Publisher's Note: All claims expressed in this article are solely those of the authors and do not necessarily represent those of their affiliated organizations, or those of the publisher, the editors and the reviewers. Any product that may be evaluated in this article, or claim that may be made by its manufacturer, is not guaranteed or endorsed by the publisher.

Copyright (C) 2022 Chen, Zhu, Hou, Yang and Chu. This is an open-access article distributed under the terms of the Creative Commons Attribution License (CC BY). The use, distribution or reproduction in other forums is permitted, provided the original author(s) and the copyright owner(s) are credited and that the original publication in this journal is cited, in accordance with accepted academic practice. No use, distribution or reproduction is permitted which does not comply with these terms. 